\title{
The successful treatment of coprophagia in resistant schizophrenia: A case report
}

D Jayakodi, S R Perera

\section{Abstract}

Coprophagia is a condition described as the compulsive consumption of faeces. It is an uncommon manifestation associated with several underlying mental health conditions. We present the case of a 32-year old male, diagnosed to have schizophrenia and on treatment with clozapine, who developed coprophagia in response to command auditory hallucinations. His condition responded to a combination of pharmacological and psychological therapy.

Key words: copraphagia, schizophrenia, clozapine, cognitive behavioral therapy (CBT)

SL J Psychiatry 2021; 12(1): 39-40

\section{Introduction}

Coprophagia is a condition described as the compulsive consumption of faeces. The word, stems from Greek: "copros" and "phagein" meaning "faeces" and "to eat" respectively (1). Several psychiatric disorders including dementia, autism, and obsessive-compulsive disorder, have been associated with coprophagia (2). However, there are only a few reported cases of cophrophagia occurring in schizophrenia (3).

Given the potential for serious complications due to coprophagia, several interventions (both pharmacological and behavioral) have been reported. Pharmacological options include second-generation antipsychotics, mood stabilizers and Selective Serotonin Reuptake Inhibitors (SSRIs) (4-5). Non-pharmacological strategies are based on behavioral theories and cognitive behavioural therapies (CBT) $(6,7)$. We present the successful management of coprophagia in a patient with resistant schizophrenia.

\section{Case Report}

Mr. M. is a 32-year old male from Gampola, a suburb of Kandy. He had been on treatment for schizophrenia for the past 15 years and had been on a daily dose of $100 \mathrm{mg}$ of clozapine for the past 2 months, due to resistance of symptoms to other antipsychotics. There was no evidence to suggest a learning disability.

He was admitted to the psychiatry ward due to a recent exacerbation of his symptoms. He reported that two known persons were monitoring him and controlling his emotions and activities from their home in Kelaniya, through a device implanted underneath his scalp. He said that he often heard them discussing about him, referring to him in the third person.

Coprophagia was noted during the first day of his current admission, with the staff observing him to be defecating and consuming his faeces multiple times during the day. When asked about this, he reported that he ate his own faeces in a response to the voices which commanded him to do so. Though undisclosed thus far, this behavior had been occurring for the past 3 years. Although the voices never threatened him, he had felt compelled to carry out the command, as it caused him unbearable distress if he did not do so. He said that eating the faeces gave him a temporary relief from this distress. Routine investigations, including biochemical and hematological investigations were normal.

The unusual presentation and complexity of his symptoms made managing this patient a challenge. There was an urgent need to control his coprophagia, because the command hallucination were causing him great distress and the only way he knew to reduce this distress was by eating his faeces. Therefore, we felt that his symptom of coprophagia had a compulsive nature to it, although the thought was secondary to a command hallucination and not an obsession. He did not have any obsessions or compulsions at the onset of this symptoms, nor during previous exacerbations.

The dose of clozapine was increased gradually to $300 \mathrm{mg}$. He was also prescribed sertraline and the dose was 
titrated up to $150 \mathrm{mg}$. The sertraline was prescribed in order to reduce his distress and the compulsive nature of the behaviour. He also underwent cognitive behaviour therapy, where the therapist encouraged him to recognize the hallucination as a voice, while encouraging him not to take part in the action of ingesting the faeces. Following almost six weeks of hospital stay, which included bi-weekly sessions of psychotherapy, together with the combination of clozapine and sertraline, his coprophagia had ceased. He is currently being followed up as an out-patient. Although he continues to have some hallucinations at the time of writing, he is not distressed by them and is able to distract himself with adaptive coping strategies.

The patient gave informed consent for the publication of the findings in this case report.

\section{Discussion}

Coprophagia in rare in schizophrenia though it is cited in the literature (5). There is no consensus on its management, but there are reports of improvement of symptoms with the addition of a SSRI (5).

Our patient's behaviour of eating his faeces stemmed from the command auditory hallucinations that he experienced. Auditory hallucinations in schizophrenia successfully responding to cognitive behavior therapy CBT have been reported previously, where the CBT is used as an augmentation to treatment with antipsychotics $(6,7)$. The success of CBT stems from the reduction of the catastrophic appraisals, which reduces the anxiety and distress, as well as enhancing new coping strategies (8).

The combination of the SSRI and the ten sessions of cognitive behavioral therapy appeared to have reduced our patient's anxiety and distress. It also could have helped him gain some insight, which led him to avoid the compulsion to eat his faeces.

There are case reports of secondary obsessive compulsive symptoms occurring in the course of schizophrenia, due to second generation antipsychotics, most importantly clozapine (9). However our patient had the compulsive eating of faeces prior to being started on clozapine. He had this behaviour previously while being treated with other antipsychotics, including haloperidol and olanzapine.

\section{Conclusions}

Coprophagia is an uncommon manifestation associated with several underlying mental health conditions, schizophrenia being one of them. Due to the lack of guidelines on the management of this condition, clinicians need to customize the management plan according to the underlying psychopathology and other clinical features.

\section{Acknowledgments}

The authors acknowledge the patient and his family, for their support for the preparation of this case report.

\section{Conflicts of interest}

None declared.

D Jayakodi, Psychiatry Unit, Teaching Hospital, Peradeniya, Sri Lanka

S R Perera, Department of Psychiatry, Faculty of Medicine, University of Peradeniya and Psychiatry Unit, Teaching Hospital, Peradeniya, Sri Lanka

Corresponding author: S R Perera

Email: sayuperera@yahoo.com

http://orcid.org/0000-0001-6824-8991

\section{References}

1. Azizi H, Khan T, Canale C, et al. The Pathophysiology and Management of Coprophagia: A Report of Two Cases and Literature Review. Case Rep Psychiatry 2018; 2018: 5157879 .

2. Josephs KA, Whitwell JL, Parisi JE, Lapid MI. Coprophagia in neurologic disorders. J Neurol 2016; 263(5): 1008-14.

3. Chaturvedi SK. Coprophagia in a schizophrenic patient: case report. Psychopathology 1988; 21(1): 31-33.

4. Beck DA, Frohberg NR. Coprophagia in an Elderly Man: A Case Report and Review of the Literature. Int J Psychiatry Med 2005; 35(4): 417-27.

5. Bacewicz A, Martin K. Coprophagia in an 8-Year-Old Hospitalized Patient: A Case Report and Review of the Literature. Case Rep Psychiatry 2017; 2017: 6565096.

6. van der Gaag M, Valmaggia LR, Smit F. The effects of individually tailored formulation-based cognitive behavioural therapy in auditory hallucinations and delusions: a meta-analysis. Schizophr Res 2014; 156(1): 30-7.

7. Sommer IE, Slotema CW, Daskalakis ZJ, Derks EM, Blom JD, van der Gaag M. The streatment of hallucinations in schizophrenia spectrum disorders. Schizophr Bull 2012; 38(4): 704-14.

8. Schirmbeck F, Zink M. Clozapine-induced obsessivecompulsive symptoms in schizophrenia: a critical review. Curr Neuropharmacol 2012; 10(1): 88-95. 